\title{
The nature of the star clusters ESO 93-SC08 and ESO 452-SC11 ${ }^{\star}$
}

\author{
E. Bica ${ }^{1}$, S. Ortolani ${ }^{2}$, and B. Barbuy ${ }^{3}$ \\ 1 Universidade Federal do Rio Grande do Sul, Dept. de Astronomia, CP 15051, Porto Alegre 91500-970, Brazil \\ ${ }^{2}$ Università di Padova, Dept. di Astronomia, Vicolo dell'Osservatorio 5, I-35122 Padova, Italy \\ 3 Universidade de São Paulo, CP 3386, São Paulo 01060-970, Brazil
}

Received November 13, 1998; accepted February, 1999

\begin{abstract}
We present $V$ and $I$ colour-magnitude diagrams of the star clusters ESO 93-SC08 and ESO 452-SC11. Both clusters are poorly populated, and the colourmagnitude diagrams show that ESO $93-\mathrm{SC} 08$ is an intermediate age open cluster, while ESO $452-\mathrm{SC} 11$ is confirmed as a globular cluster. The magnitude difference between the turnoff and the clump/horizontal branch indicates an age of $4-5$ Gyr for ESO 93-SC08, while ESO 452-SC11 has an age comparable to the average of the halo globular clusters.

ESO 93-SC08 has a reddening of $E(B-V)=0.64$ and is located at $d_{\odot} \approx 13.7 \mathrm{kpc}$ from the Sun. It is a distant open cluster in the outer disk.

ESO 452-SC11 appears to be a southern counterpart of the Palomar clusters. We derive a reddening of $E(B-$ $V)=0.58$, a distance $d_{\odot} \approx 6.5 \mathrm{kpc}$ and a metallicity $[\mathrm{Fe} / \mathrm{H}] \approx-1.5$ for ESO $452-\mathrm{SC} 08$. The deep CMD shows evidence of depletion at the lower main sequence, similarly to other poorly populated globular clusters.
\end{abstract}

Key words: globular clusters: ESO 452-SC11, open clusters: ESO 93-SC08 - HR diagram

\section{Introduction}

The star clusters ESO 93-SC08 and ESO 452-SC11 were discovered during the ESO/Uppsala $B$ sky survey catalogue confection. ESO 93-SC08 and ESO 452-SC11 were reported to have a size of $1^{\prime}$ and $2.5^{\prime}$ respectively by Holmberg et al. (1977) and Lauberts et al. (1981). Both were indicated as very obscured, the first as a globular cluster candidate and the latter as a globular cluster.

ESO 93-SC08 is projected on the Galactic disk and ESO $452-\mathrm{SC} 11$ in a bulge-halo transition region. These

\footnotetext{
* Observations collected at the European Southern Observatory - ESO, Chile.
}

two southern clusters are poorly populated resembling some Palomar clusters.

ESO 93-SC08 has coordinates $\mathrm{B}(1950) \alpha=11^{\mathrm{h}} 17^{\mathrm{m}}$ $34^{\mathrm{s}}, \delta=-64^{\circ} 56^{\prime} 48^{\prime \prime}$ and is also designated as GCL B1117-6456 (IAU notation). The Galactic coordinates are $l=293.508^{\circ}, b=-4.041^{\circ}$.

By means of the bright giants method Webbink (1985) estimated a horizontal branch $(\mathrm{HB})$ level of $V_{\mathrm{HB}}=22.0$, which he marked as very uncertain. He also provides a reddening of $E(B-V)=0.79$, estimated from the modified cosecant law, and a distance from the Sun of $d_{\odot}=$ $59.5 \mathrm{kpc}$.

Although poorly populated, the cluster structure is rather compact, and from the core and limiting radii provided by Webbink (1985), a concentration parameter $c=1.85$ can be estimated.

No colour-magnitude diagram (CMD) is available for the cluster. Besides, after 20 years of its discovery, it is still not clear whether ESO 93-SC08 is a globular or an open cluster.

ESO 452-SC11 (GCL 1636-2818) has B(1950) $\alpha=16^{\mathrm{h}}$ $36^{\mathrm{m}} 18^{\mathrm{s}}, \delta=-28^{\circ} 18^{\prime} 00^{\prime \prime}$ and is located at $l=351.912^{\circ}$, $b=+12.07^{\circ}$.

The cluster is loose but it has a small core. A concentration parameter of $c=0.95$ can be estimated from Webbink's (1985) data.

Based on an unpublished CMD reported by Webbink (1985) an HB level of $V_{\mathrm{HB}}=16.66$ was given, and from the HI column density plus galaxy counts method a reddening of $E(B-V)=0.31$, implying a distance from the Sun $d_{\odot}=10.3 \mathrm{kpc}$. He presents a metallicity estimate of $[M / H]=-1.01$. Harris's (1996) compilation reported $E(B-V)=0.50, V_{\mathrm{HB}}=16.66$ and a distance $d_{\odot}=$ $7.3 \mathrm{kpc}$.

Minniti et al. (1995) provided an infrared CMD in $K$ vs. $(J-K)$, where the HB is probably detected at the limit of the photometry. They pointed out that the locus of the red giant branch (RGB) is consistent with $E(B-V)$ $=0.70$, assuming Webbink's (1985) metallicity value. 
In the present work the $V, I$ CMDs of these clusters are studied, motivated by the fact that they are underpopulated, and probable southern Palomar-type clusters. Another such example is E3 which shows a depleted lower main sequence (MS) above the photometric observational threshold (McClure et al. 1985; Gratton \& Ortolani 1987).

In Sect. 2 the observations are described. In Sects. 3 and 4 we discuss respectively ESO 93-SC08 and ESO 452-SC11. Finally, the concluding remarks are provided in Sect. 5.

\section{Observations and reductions}

ESO 93-SC08 and ESO 452-SC11 were observed at ESO (La Silla) with the $3.55 \mathrm{~m}$ NTT and $1.54 \mathrm{~m}$ Danish telescopes.

The SUSI camera was employed at the NTT; it consists of a $1024 \times 1024$ thinned Tektronix CCD ESO \# 17 at the Nasmyth focus $B$. The pixel size is $24 \mu \mathrm{m}\left(0.13^{\prime \prime}\right.$ on the sky), and the frame size is $2.2^{\prime} \times 2.2^{\prime}$. We show in Fig. 1 a NTT $V$ image of ESO 93-SC08 and in Fig. 2 a NTT $V$ image of ESO 452-SC11. They are both loose clusters, such as Palomar globular clusters or old open clusters.

The Danish observations of 1986 June were carried out with the $512 \times 320$ RCA ESO CCD \# 1, with pixel size $30 \times 30 \mu \mathrm{m}\left(0.47^{\prime \prime}\right)$, giving a field size $4.0^{\prime}$ vs. $2.5^{\prime}$.

The Danish 1994 May observations used the $1024 \times$ 1024 Tektronix CCD \# 28, with pixel size $24 \mu \mathrm{m}$, corresponding to $0.37^{\prime \prime} /$ pixel. The full field is $6.3^{\prime} \times 6.3^{\prime}$.

The Danish 1998 observations have been carried out using the DFOSC with the tinned $2048 \times 2048$ pixel CCD Loral/Lesser C1W7. The pixel size is $15 \mu \mathrm{m}$, equivalent to $0.39^{\prime \prime}$ on the sky, with a full field of $13^{\prime} \times 13^{\prime}$.

The $\log$ of observations is given in Table 1 .

The stellar photometry was made with the DAOPHOT II code in MIDAS environment. Landolt (1983) standard stars were used for the early observations and also Landolt (1992) for the recent ones.

For the 1986 June observations the transformation equations are

$$
\begin{aligned}
& V=25.0+0.09(B-V)+v \\
& B-V=0.66+1.075(b-v)
\end{aligned}
$$

where coefficients are for $15(V)$ and $30(B)$ s exposures and airmass of 1.15 .

The zero-point calibrations for the 1994 May 17 NTT observations, which had excellent seeing, but poor photometric quality, were derived from the Danish observations, whereas the colour terms were derived from NTT data of previous nights. For ESO 452-SC11 the Danish 1994 May data were used. The reductions were carried out in the standard way, and the calibration equations are:

$$
\begin{aligned}
& V=26.91+0.04(V-I)+v \\
& I=26.09+i
\end{aligned}
$$

where coefficients are for $30 \mathrm{~s}$ exposures and airmass of 1.1 .
For the NTT data of ESO 93-SC08 the Danish 1986 June and Danish 98 July calibrations were used.

The calibration equations for the Danish 1998 observations are:

$V=24.25+v \pm 0.02$

$I=23.04+i \pm 0.02$.

The coefficients are for $1 \mathrm{~s}$ integration. The results are similar to Danish $1986 V$ calibration within 0.04 mag. A check of the $V-I$ colour could be done following Munari \& Carraro (1996) - see their Fig. 2 and Eq. (23), which allows a transformation from $(B-V)$ to $(V-I)$ for MS stars. For such purpose, we selected 10 bright disk MS stars with $16<V<19$ (Fig. 3a). The results confirm the $(V-I)$ Danish calibration within $0.04 \mathrm{mag}$.

The main source of error in the zero point accuracy $( \pm 0.03 \mathrm{mags})$ is the magnitude transfer from the cluster images to the standard stars which can amount to 0.05 mags. An additional error source affecting observations obtained with the early CCD ESO \# 1 was due to the charge transfer inefficiency, causing a slight elongation of the stars in the east-west direction.

The photometric errors at the Danish 1986 run are approximately constant to $V \approx 19.5$ and $B \approx 21$, whereas at the Danish 1994 run they are approximately constant to $V \approx 19$ and $I \approx 18$. They amount to $0.02 \mathrm{mag}$, and increase for fainter magnitudes.

At the NTT the errors of $\approx 0.02-0.03 \mathrm{mag}$ are constant to $V \approx 22.5$ and $I \approx 22.5$, increasing for fainter magnitudes.

We also present a high quality CMD of NGC 6752 that we use for comparison purposes. The diagram is a combination of that previously published in Rosino et al. (1997), to a deeper one obtained under excellent seeing conditions, $5^{\prime}$ offcenter. The calibration was carried out using standard stars observed in the same night, which was photometric.

\section{The open cluster ESO 93-SC08}

\subsection{Colour-magnitude diagrams}

In Figs. 3a and $\mathrm{b}$ we show respectively the NTT CMDs corresponding to whole frame and an extraction of $r<$ $33^{\prime \prime}$. In Fig. 3a a bright disk MS is present, whereas in Fig. 3b the cluster sequences become clearer. The red clump, typical of intermediate age clusters, is located at $V=18.6$ and $(V-I)=1.85$. A few bright giants are detected above the clump. The cluster TO is reached as well as $\approx 2$ magnitudes of MS. The CMD of ESO 93-SC08 appears to be rather similar to that of M 67 (e.g. Demarque et al. 1992).

\subsection{Age}

The magnitude difference between the TO and the clump is an age indicator (Janes \& Phelps 1994). We have 


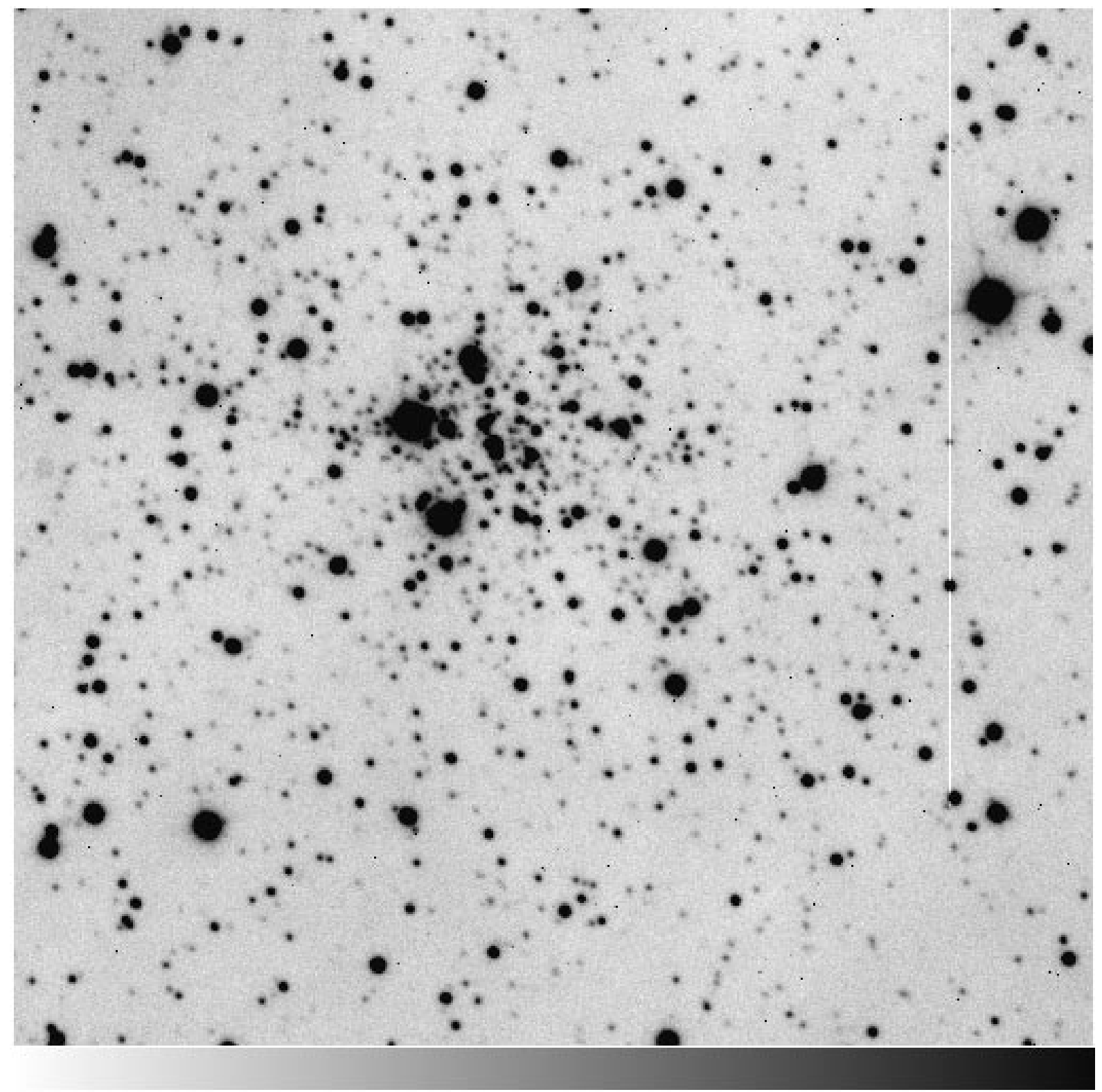

Fig. 1. NTT $V$ image ESO 93-SC08. Dimensions are $2.2^{\prime} \times 2.2^{\prime}$

measured in Demarque et al.'s (1992) CMDs this value for the reference intermediate age clusters M 67 and NGC 188, resulting $\Delta V_{\text {clump }}^{\mathrm{TO}}=2.2$ and 2.75 respectively. For ESO 93-SC08 we obtain $\Delta V_{\text {clump }}^{\mathrm{TO}}=2.3 \pm 0.20$ (Fig. $3 \mathrm{~b}$ ). Adopting Demarque et al.'s ages of 4 Gyr (M 67) and 6.5 Gyr (NGC 188), there results $4-5$ Gyr for the present cluster. Therefore ESO 93-SC08 adds to the list of the about 20 old open clusters with ages $>4$ Gyr (Friel 1995; Carraro et al. 1998).

\subsection{Reddening and distance}

We take as reference point in the CMD the dereddened locus of the clump in intermediate age clusters; Garnavich et al. (1994) indicates $M_{V}=0.94$ and $(V-I)_{0}=1.0$ for M 67. With values for ESO 93-SC08 from Sect. 3.1 we obtain $E(V-I)=0.85$, which converts to $E(B-V)=$ 0.64 (Dean et al. 1978) for ESO 93-SC08. This reddening value is larger than that obtained from the integrated spectrum by Bica et al. (1998), where there is probably an effect of less reddened foreground stars contaminating this poorly populated cluster, and also the integrated light must be affected by statistical fluctuations owing to the small number of bright member stars.

Adopting a selective-to-total absorption of $R=3.1$, we get $A_{V}=1.98$. An absolute distance modulus of $(m-$ $M)_{0}=15.68 \pm 0.2$ and a distance from the Sun $d_{\odot}=$ $13.5 \pm 0.6 \mathrm{kpc}$. 


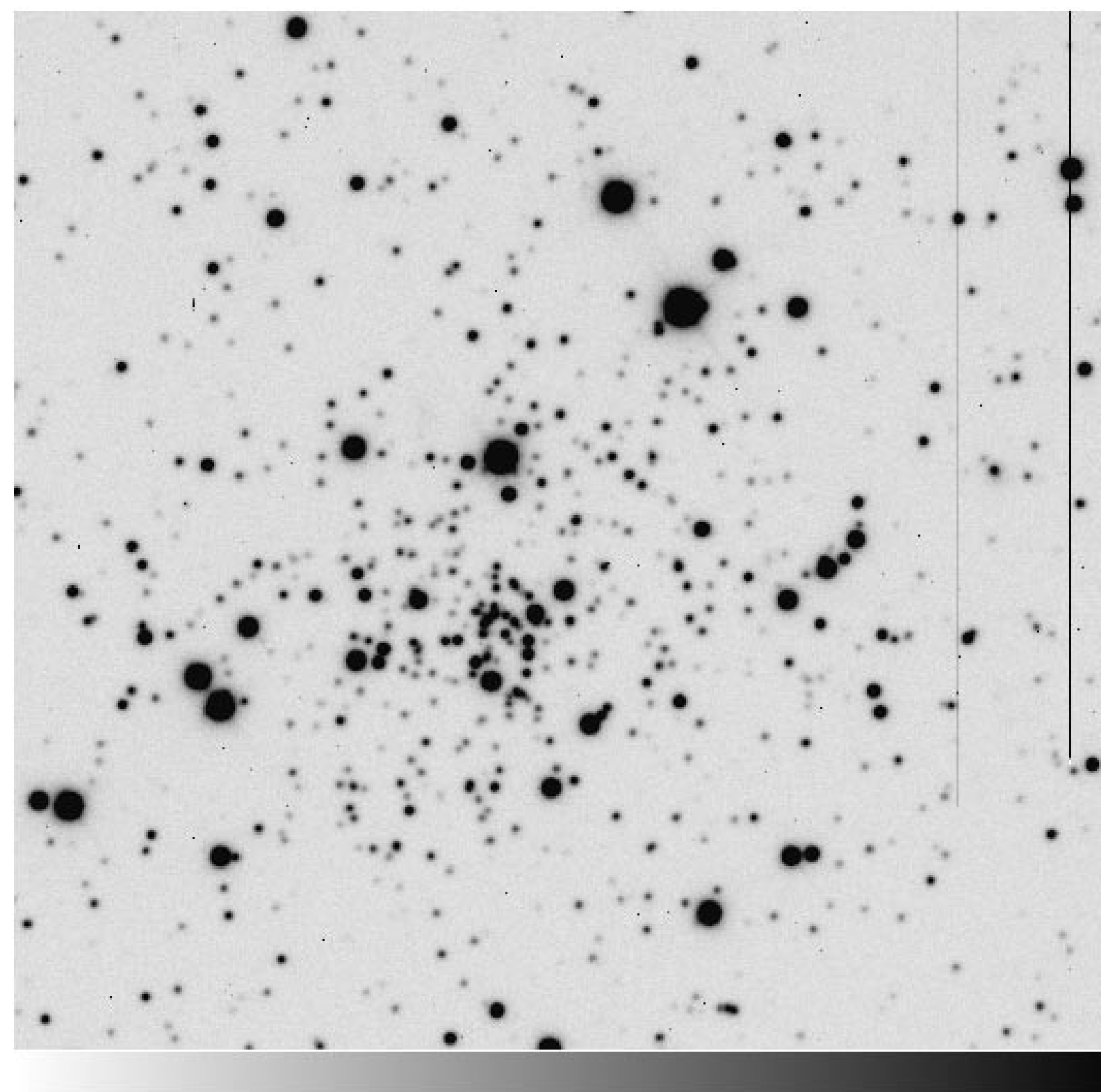

Fig. 2. NTT $V$ image ESO 452-SC11. Dimensions are $2.2^{\prime} \times 2.2^{\prime}$

The Galactocentric coordinates of the cluster, assuming a distance of the Sun to the Galactic center of $R_{\odot}=8.0 \mathrm{kpc}$ (Reid 1993), are $X=-2.6(X<0$ refers to our side of the Galaxy), $Y=-12.5 \mathrm{kpc}$ and $Z=$ $-1.0 \mathrm{kpc}$. The galactocentric distance projected on the plane is $r=12.8 \mathrm{kpc}$, indicating that this is probably the most distant open cluster relative to the Galactic center.

The location of the cluster with respect to the open clusters older than 2 Gyr is shown in Fig. 4, where the data given in Friel (1995) are adopted, using a distance to the Galactic center of $8 \mathrm{kpc}$ (Reid 1993). ESO 93-SC08 is among the farthest, and is also among the open clusters more distant from the Galactic plane. Such outlyers may occur as far as the disk border in HI (Kulkarni et al. 1982), HII regions (Flich \& Blitz 1984), and luminous stars
(Moffat et al. 1979), which point to an extent of the disk of $20-30 \mathrm{kpc}$.

It is also interesting to discuss the galactic location of old open star clusters and three metal rich globular clusters with a structure similar to that of open clusters. Two of them were until recently classified as open clusters, but analyses by means of CMDs, spectroscopy of individual stars and integrated spectroscopy, point to a globular cluster nature: Lynga 7 (Ortolani et al. 1993; Tavares \& Friel 1995), and BH 176 (Ortolani et al. 1995a). Besides, the globular cluster candidate Palomar 10, which has a loose structure, shows a CMD compatible with that of a metal-rich globular cluster (Kaisler et al. 1997). Integrated spectroscopy of Lynga 7, BH 176 and Palomar 10, which are all projected on the disk, indicates that they are 

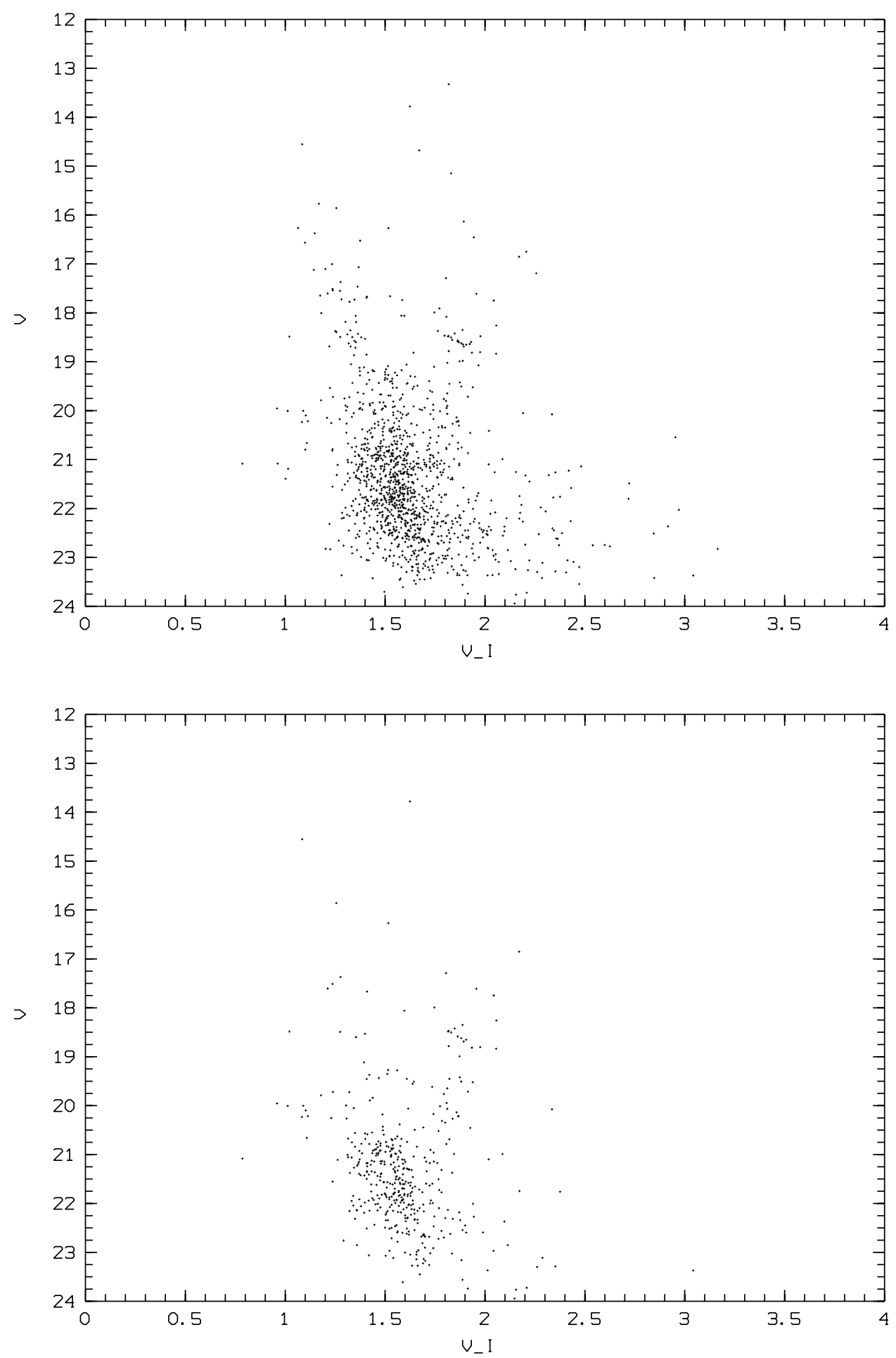

Fig. 3. ESO 93-SC08: $V$ vs. $(V-I)$ CMD for the a) NTT whole frame; b) $r<33^{\prime \prime}$ 
Table 1. Log of observations

\begin{tabular}{|c|c|c|c|c|c|}
\hline Target & Filter & Date & Tel. & $\begin{array}{l}\text { Exp. } \\
(\mathrm{sec})\end{array}$ & $\begin{array}{l}\text { Seeing } \\
\left({ }^{\prime \prime}\right)\end{array}$ \\
\hline \multirow{9}{*}{ ESO 93-SC08 } & $V$ & \multirow{3}{*}{23.6 .95} & \multirow{3}{*}{ NTT } & 420 & 0.8 \\
\hline & $V$ & & & 60 & 0.6 \\
\hline & $I$ & & & 300 & 0.6 \\
\hline & $B$ & \multirow[t]{2}{*}{01.6 .86} & \multirow[t]{2}{*}{ Danish } & 480 & 1.4 \\
\hline & $V$ & & & 300 & 1.4 \\
\hline & $V$ & \multirow[t]{4}{*}{03.7 .98} & \multirow[t]{4}{*}{ Danish } & 60 & 1.4 \\
\hline & $V$ & & & 60 & 1.4 \\
\hline & $I$ & & & 60 & 1.4 \\
\hline & $I$ & & & 60 & 1.4 \\
\hline \multirow[t]{6}{*}{ ESO 452-SC11 } & $V$ & \multirow[t]{4}{*}{17.5 .94} & \multirow[t]{4}{*}{ NTT } & 600 & 0.85 \\
\hline & $I$ & & & 60 & 0.85 \\
\hline & $I$ & & & 60 & 0.8 \\
\hline & $I$ & & & 600 & 0.70 \\
\hline & $V$ & \multirow[t]{2}{*}{20.5 .94} & \multirow[t]{2}{*}{ Danish } & 60 & 1.4 \\
\hline & $I$ & & & 60 & 1.4 \\
\hline \multirow[t]{2}{*}{ NGC 6752} & $V$ & 16.5 .94 & NTT & 300 & 0.6 \\
\hline & $I$ & 16.5.94 & NTT & 300 & 0.6 \\
\hline
\end{tabular}

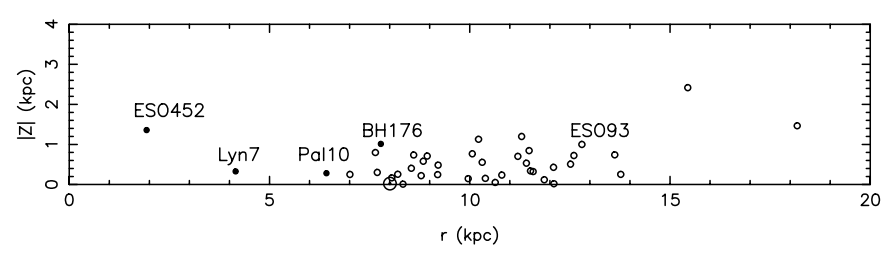

Fig. 4. Distance from the plane $|z|$ vs. Galactocentric radial distance projected on the plane $r$ for known open clusters of ages $>2$ Gyr (Friel 1995) and ESO 93-SC08 (open circles), where are also plotted ESO 452-SC11, besides Lynga 7, Palomar 10 and $\mathrm{BH} 176$ (classified as globulars) (filled circles)

similar to metal-rich globular clusters (Bica et al. 1998). In Fig. 4 are also indicated the positions in the Galaxy of these three objects.

\section{The globular cluster ESO 452-SC11}

\subsection{Colour-magnitude diagrams}

For comparison purposes, we present in Fig. 5 a very deep CMD of the intermediate metallicity reference cluster NGC 6752 , to be used as template. Notice the MS extending along 6 magnitudes.

In Fig. 6 a is shown the whole frame $V$ vs. $(V-I)$ NTT CMD for ESO 452-SC11, which is clearly dominated by the cluster features. The MS extends to $V \approx 23$, about 3 magnitudes below the TO. A poorly populated giant branch is present together with an HB which contains a red component, and some bluer stars.

In Fig. $6 \mathrm{~b}$ is shown an extraction of $r<65^{\prime \prime}$ where the mean locus of NGC 6752 is overplotted.

In Fig. 6c we show a Danish CMD corresponding to an extraction of $r<94^{\prime \prime}$, a slightly larger area than that presented in Fig. 6a. In this sampling there appear two

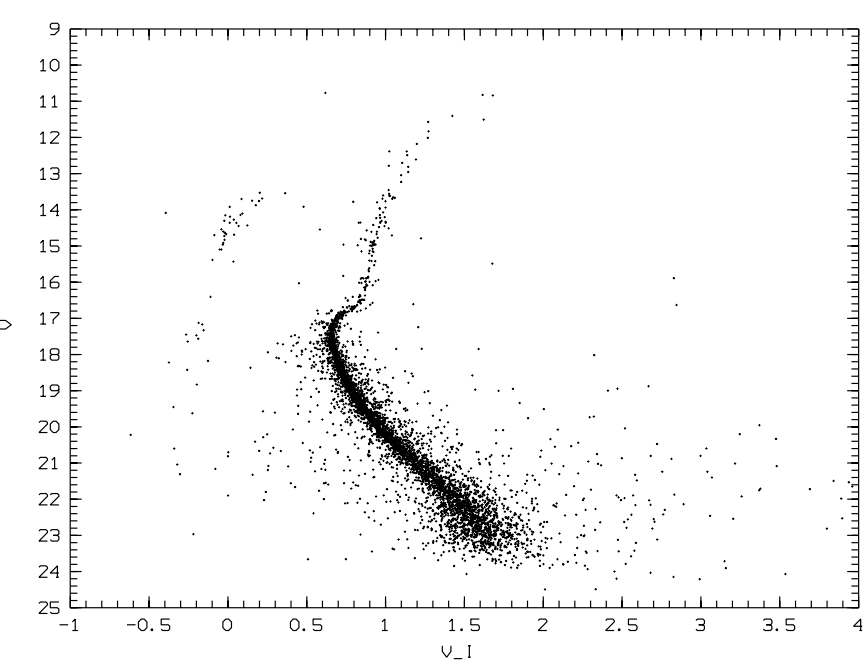

Fig. 5. NGC 6752: $V$ vs. $(V-I)$ CMD which is a combination of bright sequences (Rosino et al. 1997) and the present data containing a deep main sequence

additional bright giants, and one probable asymptotic giant branch (AGB) star.

In Fig. 7 the field surrounding the cluster is given, corresponding to $r>188^{\prime \prime}$ (Danish frame). Since the cluster Galactic latitude is only $b=+12.07^{\circ}$, a disk field contamination is not unexpected. In fact, a field MS extends along $16.5<V<19.0$. Below this the cluster TO is evident, which is somewhat bluer than the field stars. This shows that cluster stars are still detected at such radii.

\subsection{Cluster metallicity}

The morphology of the HB is not purely red, indicating that this is not a metal-rich cluster like 47 Tuc. In Fig. $6 \mathrm{~b}$ is shown the mean locus of NGC 6752 (Rosino et al. 1997) overplotted on the NTT extraction of the cluster. The RGB and subgiant branch are well matched. Although the HB morphology is different, a metallicity similar to that of NGC $6752([\mathrm{Fe} / \mathrm{H}]=-1.54$, Zinn 1985) can be assigned to ESO 452-SC11. Webbink (1985) gives $[\mathrm{Fe} / \mathrm{H}] \approx-1.0$, probably based on the HB morphology.

\subsection{Reddening and distance}

We calculate the cluster reddening taking NGC 6752 as reference. The $(V-I)$ colour of the RGB at the HB level for ESO $452-\mathrm{SC} 11$ is $1.66 \pm 0.03$, whereas for NGC 6752 it is $(V-I)=0.95 \pm 0.04$, giving a difference of $\Delta(V-I)=0.71$. Adopting $E(B-V)=0.04$ for NGC 6752 (Zinn 1980), and $E(V-I) / E(B-V)=1.31$ (Dean et al. 1978), we get $E(V-I)=0.76$ which converts to $E(B-V)=0.58$ and $A_{V}=1.80\left(R=A_{V} / E(B-V)=\right.$ 3.1, Savage \& Mathis 1979) for ESO 452-SC11.

The HB level in ESO $452-\mathrm{SC} 11$ is located at $V_{\mathrm{HB}}=$ $16.55 \pm 0.15$. The $\mathrm{HB}$ absolute magnitude for $[\mathrm{Fe} / \mathrm{H}]=$ 

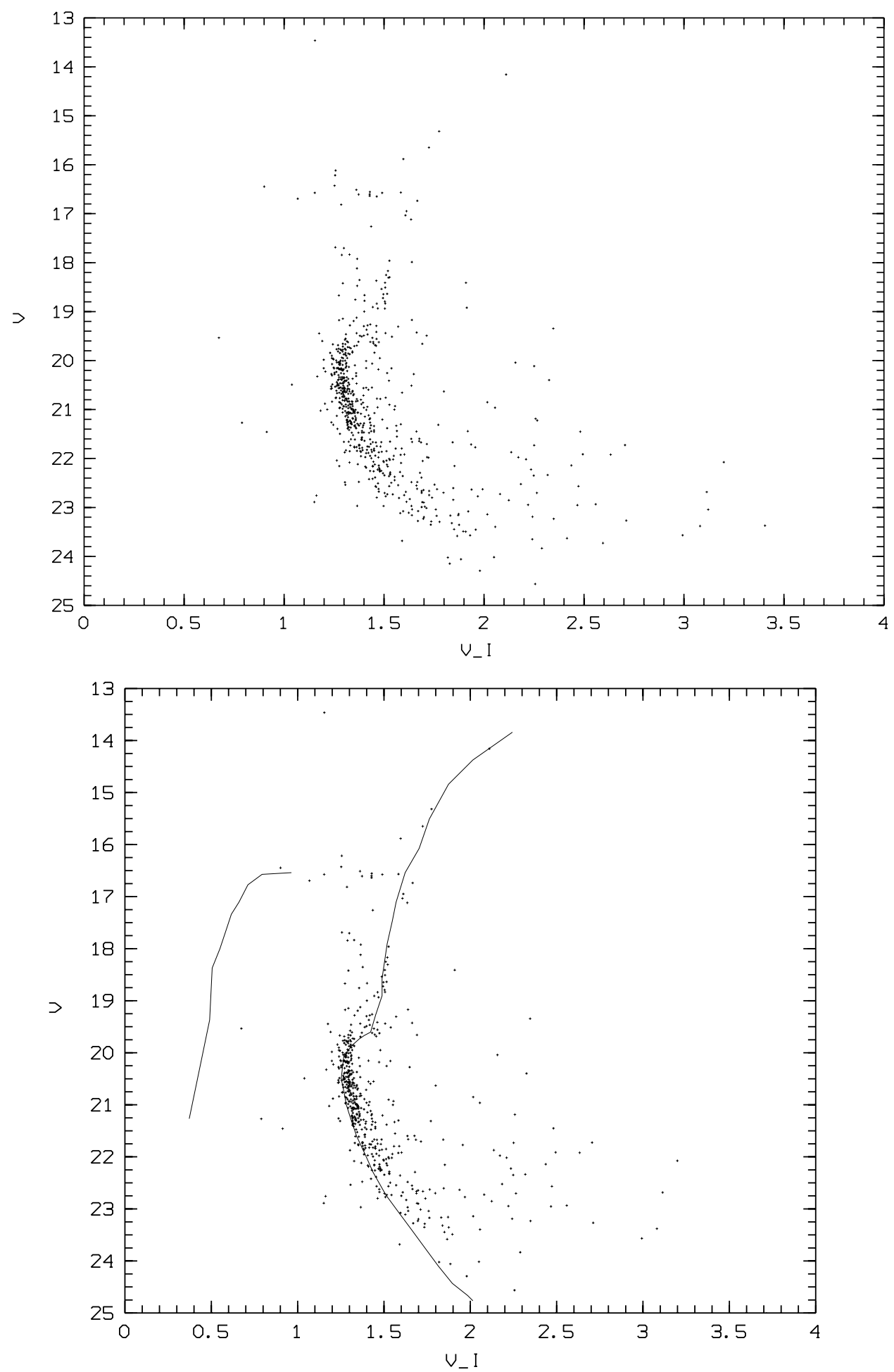

Fig. 6. ESO 452-SC11: $V$ vs. $(V-I)$ NTT CMD for a) whole frame; b) extraction of $r<65^{\prime \prime}$ around the cluster center, where the mean locus of NGC 6752 is overplotted; c) Danish CMD with an extraction $r<94^{\prime \prime}$ 


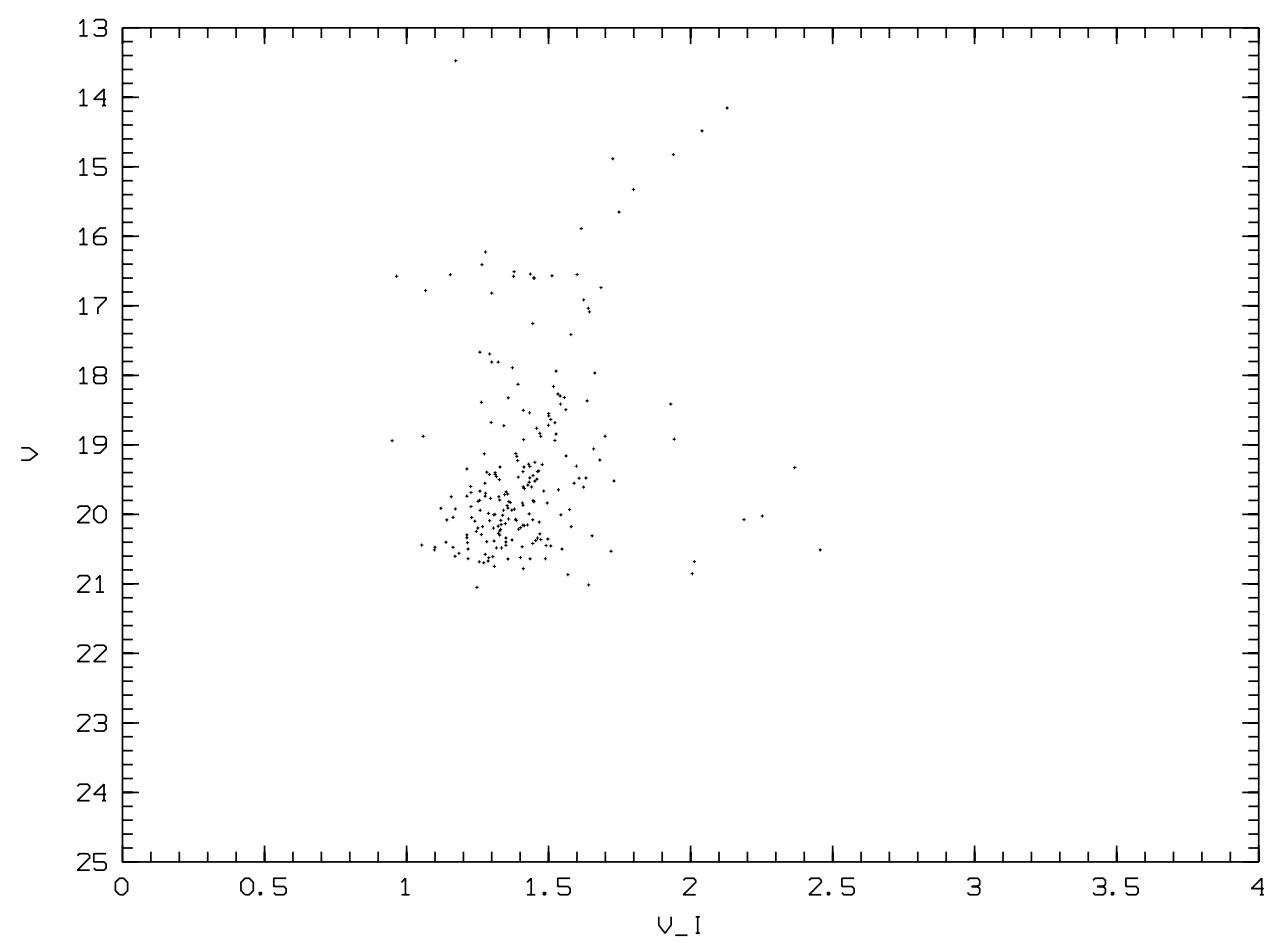

Fig. 6. continued

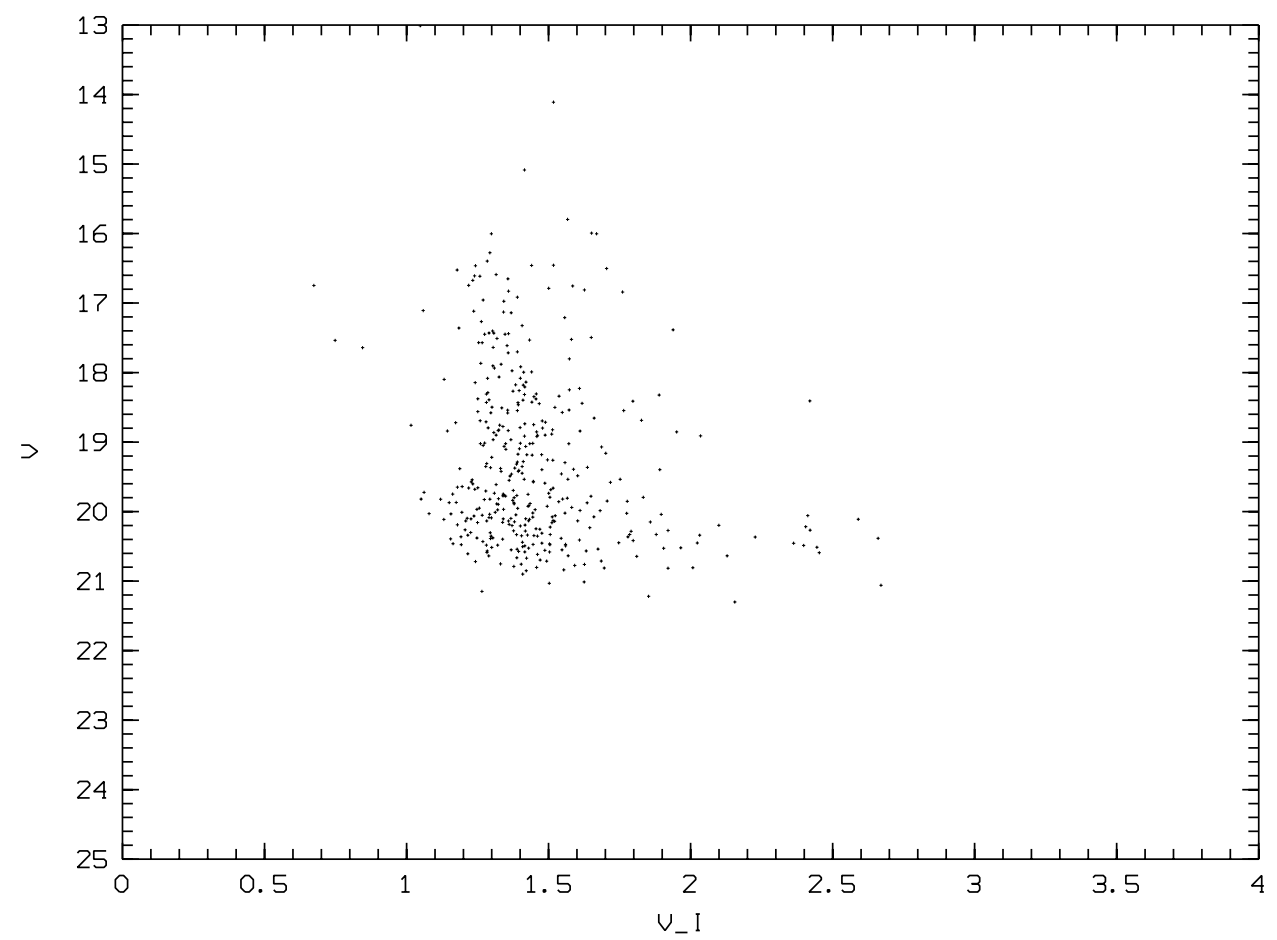

Fig. 7. Field around ESO 452-SC11, for $r>188^{\prime \prime}$ (Danish frame) 


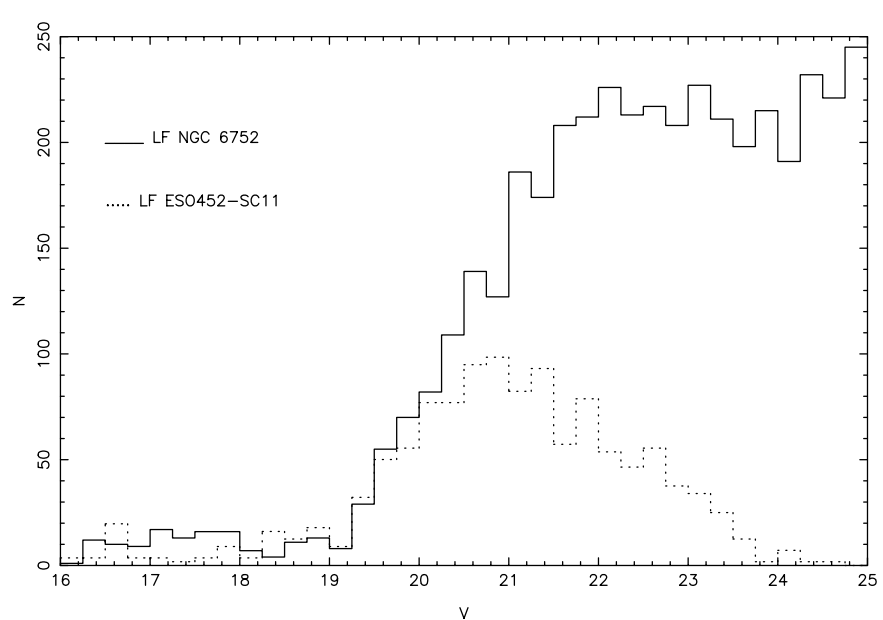

Fig. 8. Luminosity function of ESO 452-SC11 (dotted lines) compared to that for NGC 6752 (solid lines). Notice the depletion at fainter magnitudes for ESO 452-SC11

-1.5 is $M_{V}=0.7$ (Buonanno et al. 1989; Lee et al. 1990; Sandage \& Cacciari 1990), and there results an absolute distance modulus of $(m-M)_{0}=14.05 \pm 0.25$, and a distance of $d_{\odot}=6.5 \pm 0.7 \mathrm{kpc}$.

The Galactocentric coordinates of the cluster, assuming a distance of the Sun to the Galactic center of $R_{\odot}=8.0 \mathrm{kpc}($ Reid 1993), are $X=-1.71, Y=-0.90$ and $Z=1.36 \mathrm{kpc}$. We also show in Fig. 4 the $|\mathrm{Z}|$ vs. $r$ location of ESO 452-SC11. The cluster is at the bulge distance, but slightly above the bulk of the bulge population.

\subsection{Age}

It can be seen in Fig. 6a that the cluster CMD reaches several magnitudes below the TO. We measured a $\Delta V_{\mathrm{HB}}^{\mathrm{TO}}=3.6 \pm 0.2$, which means that this is an old globular cluster of age comparable to the average of the halo clusters, for which a mean of $\Delta V_{\mathrm{HB}}^{\mathrm{TO}}=3.6$ was found by Buonanno et al. (1989). The overplot of the mean locus of NGC 6752 , with $\Delta V_{\mathrm{HB}}^{\mathrm{TO}}=3.65$ (Buonanno et al. 1989) shows that their TO morphologies are slightly different, and that ESO 452-SC11 would be somewhat younger than this reference cluster.

ESO 452-SC11 could belong to the halo considering its age and metallicity. On the other hand, from its location in the Galaxy (Fig. 4), it could also be a bulge member at the extreme lower end of the metallicity distribution (McWilliam \& Rich 1994), in which case the old age is also compatible (Ortolani et al. 1995b).

Kinematical properties of the cluster would be necessary to decide about its subsystem membership in the Galaxy.

\subsection{Depletion of low mass stars?}

It appears from the CMD (Figs. 6) that ESO 452-SC11 shows a depopulated MS, as compared to the reference cluster NGC 6752 (Fig. 5). In Fig. 8 we present the luminosity function of ESO 452-SC11 compared to that of NGC 6752, where the numbers for ESO 452-SC11 are normalized to those for NGC 6752 in the interval $18<V<$ 20. Notice a drop for $V>20.5$. We carried out artificial stars experiments, in order to check the completeness by counting retrieved stars. The results show a $100 \%$ completeness at $V<20.6$, going down to $84 \%$ at $V=21.0$, and to $81 \%$ at $V=22.0$. The last bin at $V=23.0$ shows $77 \%$. These completeness corrections cannot account for the drop in the luminosity function, which appears to be significantly affected by low mass stars segregation. The stellar mass corresponding to the drop in the luminosity function is close to the turnoff mass. We might be facing a case of a cluster evaporation, similarly to that of E3 (see Veronesi et al. 1996 for a recent discussion). Some evidence of this was also found for NGC 6717 (Palomar 9) see Ortolani et al. (1999).

\section{Conclusions}

We studied the loose star clusters ESO 93-SC08 and ESO $452-\mathrm{SC} 11$ in $V, I$ CMDs. ESO 93-SC08 is an intermediate age cluster, located at $d_{\odot} \approx 13.7 \mathrm{kpc}$ from the Sun, it is thus part of the outer disk.

ESO 452-SC11 is confirmed as a globular cluster, for which we derived reliable parameters. From its location, metallicity and age, it may be a key cluster to investigate the bulge-halo transition.

Acknowledgements. BB and EB acknowledge partial financial support from $\mathrm{CNPq}$ and Fapesp.

\section{References}

Bica E., Clariá J.J., Piatti A.E., Bonatto C., 1998, A\&AS 131, 483

Buonanno R., Corsi C.E., Fusi Pecci F., 1989, A\&A 216, 80

Carraro G., Ng Y.K., Portinari L., 1998, MNRAS 296, 1045

Dean J.F., Warren P.R., Cousins A.J., 1978, MNRAS 183, 569

Demarque P., Green E.M., Guenther D.B., 1992, AJ 103, 151

Flich M., Blitz L., 1984, ApJ 279, 125

Friel E.D., 1995, ARA\&A 33, 381

Garnavich P.M., Vandenberg D.A., Zurek D.R., Hesser J.E., 1994, AJ 107, 1097

Gratton R.G., Ortolani S., 1987, A\&AS 67, 373

Harris W.E., 1996, AJ 112, 1487

Holmberg E.B., Lauberts A., Schuster H.E., West R.M., 1977, A\&AS 27, 295

Janes K.A., Phelps R.L., 1994, AJ 108, 1773

Kaisler D., Harris W.E., McLaughlin D.E., 1997, PASP 109, 920

Kulkarni S.R., Blitz L., Heiles C., 1982, ApJ 259, L63 
Landolt A.U., 1983, AJ 88, 439

Landolt A.U., 1992, AJ 104, 340

Lauberts A., Holmberg E.B., Schuster H.E., West R.M., 1981, A\&AS 43, 307

Lee Y.-W., Demarque P., Zinn R., 1990, ApJ 350, 155

McClure R.D., Hesser J.E., Stetson P.E., Stryker L.L., 1985, PASP 97, 665

McWilliam A., Rich R.M., 1994, ApJS 91, 749

Minniti D., Olszewski E.W., Rieke M., 1995, AJ 110, 1686

Moffat A.F.J., Fitzgerald M.P., Jackson P.D., 1979, A\&AS 38, 197

Munari U., Carraro G., 1996, A\&A 314, 108

Ortolani S., Barbuy B., Bica E., 1999, A\&A (in press)

Ortolani S., Bica E., Barbuy B., 1993, A\&A 273, 415

Ortolani S., Bica E., Barbuy B., 1995a, A\&A 300, 726

Ortolani S., Renzini A., Gilmozzi R., et al., 1995b, Nat 377, 701
Reid M., 1993, ARA\&A 31, 345

Rosino L., Ortolani S., Barbuy B., Bica E., 1997, MNRAS 289, 745

Sandage A., Cacciari C., 1990, ApJ 350, 645

Savage B., Mathis J., 1979, ARA\&A 17, 73

Tavares M., Friel E.D., 1995, AJ 110, 223

Veronesi C., Zaggia S., Piotto G., Ferraro F.R., Bellazzini M., 1996, in Formation of the Galactic Halo, Inside and Out, ASP Conf. No. 92, Morrison H., Sarajedini A. (eds.), p. 301

Webbink R.F., 1985, in Dynamics of Star Clusters IAU Symp. 113, Goodman J. and Hut P. (eds.), Dordrecht: Reidel, p. 541

Zinn R., 1980, ApJS 42, 19

Zinn R., 1985, ApJ 293, 424 